Binghamton University

The Open Repository @ Binghamton (The ORB)

$1-1-2019$

\title{
Dynamics of a Threshold Shock Sensor: Combining Bi-stability and Triboelectricity
}

Daniel S. Nelson

Binghamton University--SUNY, dnelson7@binghamton.edu

Alwathiqbellah Ibrahim

Wathiq@binghamton.edu

Shahrzad Towfighian

Binghamton University--SUNY, stowfigh@binghamton.edu

Follow this and additional works at: https://orb.binghamton.edu/mechanical_fac

Part of the Mechanical Engineering Commons

\section{Recommended Citation}

D. Nelson, A. Ibrahim, S. Towfighian (2019), Dynamics of a Threshold Shock Sensor: Combining Bi-stability and Triboelectricity, Sensors and Actuators: A Physical, Vol. 285, pp. 666-675, Published by Elsevier.

This Article is brought to you for free and open access by the Mechanical Engineering at The Open Repository @ Binghamton (The ORB). It has been accepted for inclusion in Mechanical Engineering Faculty Scholarship by an authorized administrator of The Open Repository @ Binghamton (The ORB). For more information, please contact ORB@binghamton.edu. 


\title{
Dynamics of a Threshold Shock Sensor: Combining Bi-stability and Triboelectricity
}

\author{
Daniel Nelson ${ }^{1}$, Alwathiqbellah Ibrahim ${ }^{2}$, and Shahrzad Towfighian ${ }^{3}$ \\ 1,2,3 Binghamton University, 4400 Vestal Parkway East, Binghamton, NY 13902.
}

\begin{abstract}
A proof of concept of a triboelectric threshold shock sensor and its characterization are presented. Shock sensors are used in many applications in the automotive, shipping and other industries, mainly to determine if acceleration thresholds are met. Many shock sensors are only mechanical, so the only way to know if the threshold has been reached is to physically check the device. There are noticeable advantages of using triboelectric transduction and bi-stability to create a shock sensor. By combining a buckled-beam structure and a triboelectric generator, we created a proof of concept of a tunable threshold shock sensor. The sensor generates a voltage peak only if the base acceleration is beyond a threshold. In addition, the sensor produces voltage proportional to the base acceleration beyond the threshold acceleration. This means the output signal provides more information about the strength of the shock that the device experiences. The sensor concept is illustrated for a threshold shock of $3.26 \mathrm{~g}$, but the threshold can be tuned by increasing the compressive axial force of the buckled beam. Increasing this axial force increases the threshold shock the sensor can detect. Thus, the combined system is a tunable threshold shock sensor with enhanced functionality. We presented a mathematical model that captures important observations of the experiments and can be used as a design tool for more precise, high-resolution triboelectric shock sensors.
\end{abstract}

Keywords: Triboelectric, Shock Sensor, Bi-stable, Impact, Buckling, Threshold Shock, Numerical Simulation, Experimental Results.

\section{Introduction}

Shock sensors have a wide application in the auto-industry [1], recycle systems in aerospace [2], fuse systems [3], earthquake detection [4] airbag systems [5-7], weapons arming and disarming [8, 9], and senior fall detection [10]. Fast response, high sensitivity, and reliability are considered major requirements for shock sensors. Different actuation mechanisms have been investigated: mechanical [11, 12], electrostatic [13-16], electrothermal [17-19], piezoelectric [20-25], optical actuation [26] and electromagnetic [27, 28]. Among existing mechanisms, electrostatic actuators are the most common. However, they often suffer from pull-in instability. Electromagnetic transducers, another common actuation type, consume large amounts of power (in the $m W$ range). To avoid the requirement of continuous powering, mechanical buckling offers two stable, switchable states $[18,29]$. The simplest mechanical structures for buckling are bi-stable buckled beams or shallow arches that can achieve large displacements from low forces. These structures are ideal for systems that require on and off or open and closed positions because of their high speed and tunability. Harmonic excitation can switch the beam between the two stable positions $[30,31]$. A critical amount of energy introduced to the actuator activates the transition of the system between those stable points [32, 33].

Many studies have been conducted on threshold acceleration sensors, which are also referred to as gsensors in the literature. The concept of a threshold sensor is that the sensor will switch stable states when the acceleration exceeds a threshold value and will remain stationary while the acceleration is below the threshold. Acceleration sensors can be designed based on cantilever beams [34-36], clamped-clamped beams

Email address: ${ }^{1}$ dnelson7@binghamton.edu, ${ }^{2}$ Wathiq@binghamton.edu, ${ }^{3}$ corresponding author: stowfigh@binghamton.edu. (Daniel Nelson ${ }^{1}$, Alwathiqbellah Ibrahim ${ }^{2}$, and Shahrzad Towfighian ${ }^{3}$ ) 


\section{Nomenclature}

\begin{tabular}{|c|c|}
\hline$A$ & Cross-sectional area of beam \\
\hline$b, b_{m}$ & Width of beam, width of center mass and upper electrode \\
\hline$B$ & Arbitrary constant \\
\hline$c, c_{i}$ & Damping term, impact damping term \\
\hline$d_{0}$ & Initial total gap with no compressive axial load \\
\hline$D_{1}$ & Damping coefficient \\
\hline$E$ & Young's Modulus \\
\hline$E_{\text {air }}, E_{P D M S}$ & Electric field in air gap, PDMS layer \\
\hline$F_{d}, F_{s}$ & Impact damping force, impact stiffness force \\
\hline$g_{i}$ & Initial gap between upper electrode and PDMS \\
\hline$I$ & Moment of inertia \\
\hline$J$ & Jacobian \\
\hline$K_{L}, K_{Q}, K_{C}, k_{i}$ & Linear, quadratic, cubic, impact stiffness coefficients \\
\hline$L, L_{m}$ & Length of beam, length of center mass and upper electrode \\
\hline$L_{1}$ & Midspan location and location of concentrated mass \\
\hline$M$ & Concentrated mass consisting of center mass and upper electrode \\
\hline$M_{1}, M_{z}$ & Mass coefficient, base excitation mass coefficient \\
\hline$p$ & Compressive axial force \\
\hline$q(t)$ & Charge transferred \\
\hline$R$ & Load resistance \\
\hline$s$ & Constant for mode shape analysis \\
\hline$S$ & Contact area \\
\hline$t, t_{m}$ & Thickness of beam, thickness of center mass and upper electrode \\
\hline$T$ & Kinetic Energy \\
\hline$T_{P M D S}$ & Thickness of PDMS layer \\
\hline$u(x, t)$ & Longitudinal deflection of the beam \\
\hline$U$ & Potential Energy \\
\hline$v(x, t)$ & Dynamic deflection about buckled configuration \\
\hline$V$ & Volume \\
\hline$w(x, t)$ & Absolute transverse deflection of the beam \\
\hline$W_{n c}$ & Work done by the nonconservative forces \\
\hline$y(x, t)$ & Relative transverse deflection of the beam \\
\hline$z(t)$ & Base excitation \\
\hline$z_{c}$ & Transverse coordinate direction \\
\hline$\alpha, \beta$ & Constants for mode shape analysis \\
\hline$\alpha_{1,2,3}$ & Electrical constants \\
\hline$\gamma_{i}(t)$ & Time dependent function \\
\hline$\delta_{1}$ & Penetration distance \\
\hline$\varepsilon_{0}, \varepsilon_{r}$ & Vacuum permittivity, relative permittivity \\
\hline$\varepsilon_{s}$ & Mechanical strain \\
\hline$\eta(t)$ & Arbitrary time dependent function \\
\hline$\lambda, \lambda_{1}$ & Eigenvalues \\
\hline$\rho$ & Density of polymer beam \\
\hline$\sigma$ & Surface charge density \\
\hline$\sigma_{s}$ & Mechanical stress \\
\hline$\phi(x)$ & Mode shape \\
\hline$\psi(x)$ & Buckled/Static configuration \\
\hline$\omega$ & Natural frequency \\
\hline
\end{tabular}


[2], or other features [6, 37]. When sufficient force is applied, the structure moves from one stable point to another through snap-through motion. This snap-through motion usually opens or closes a circuit or sends an analog signal using a transducer to indicate the device has switched states. Triboelectric transducers are emerging as a conversion mechanism because of their great energy density and easy, low-cost fabrication $[38,39]$. They require contact and separation, which makes them useful for shock (impact) sensing. However, current triboelectric devices cannot distinguish when the shock gets past a limit. Combining triboelectric transducers with a bi-stable structure enables making threshold sensors that not only activate beyond a shock limit, but they can also provide information about the strength of the shock.

This work will demonstrate the feasibility of a threshold sensor that uses triboelectric transduction. It uses the bi-stable mechanism of a buckled clamped-clamped beam carrying a triboelectric generator at the center, see Fig. 1. Initially, a static axial compressive load buckles the straight beam to its stable, upper position. When the excitation acceleration exceeds a threshold, the buckled beam will snap to its lower stable position, and the triboelectric layers will collide. During this transition, there will be a rapid change in the potential energy of the structure that is converted to a voltage signal in the triboelectric transducer. The threshold shock can be tuned using the initial compressive load. In addition, because the voltage spike is a function of the impact, the threshold sensor can act as an accelerometer beyond the threshold shock.

Up to this point, there have not been many efforts to describe the electro-mechanical behavior of a triboelectric device in a continuous model. Therefore, one of the highlights of this paper is to shed light on the multiphysics and mathematical modeling so that this derivation can be used as a learning tool and building block for more efforts concerning sensors using triboelectric generators. So to accurately simulate the sensor behavior, a continuous electro-mechanical model based on the nonlinear Euler-Bernoulli beam theory was developed to capture the dynamical responses. This effort on the macro-prototype of the shock sensor provides a fundamental understanding of the electro-mechanical system response and a design platform for its future miniature development.

The organization of this paper is as follows. The principle of operation of the sensor will be described. Then a continuous model will be introduced, deriving the static configuration, mode shape, and reduced order model. The impact model will be described, and the experimental setup will be displayed in detail. Comparisons between theoretical and experimental trials will be shown to prove the validity of the model. The discussion ends with a conclusion.

\section{Principle of Operation}

The principle of operation of the shock sensor is shown in Figs. 1a and 1b. The figures show the upper and lower stable configurations of the threshold sensor. We increase the axial force on an initially straight beam until it buckles (Fig. 2). In the initial configuration, when the upper and lower electrodes are separated, there is a charge equilibrium, so there is no voltage across the load. Once the device is triggered, the upper electrode travels toward the lower electrode and as the gap changes, the electrical potential difference also changes. Two factors that affect the voltage potential difference are added mass at the midspan of the beam and the velocity of the upper electrode. With a larger center mass, the inertial force will be greater that will result in a higher velocity of the upper electrode. With this higher velocity, there will be a larger change in the voltage potential difference. Once the upper and lower electrodes come in contact with each other, the voltage potential difference quickly drops to zero. The voltage potential will remain at zero while the device remains at the lower configuration. Next, the mathematical model of the coupled electro-mechanical system will be developed to shed light on the system behavior.

\section{Mathematical Modeling}

Because this system includes large deformations of the beam attached to a center mass, we will develop a continuous model to accurately describe its behavior. A continuous model is chosen because: (i) it is more accurate than a lumped parameter model; (ii) the effect of the mid-plane stretching will not have to be determined experimentally; and (iii) the axial force can be easily incorporated into the model. To reduce the complexity of the continuous system, we will assume that the added center mass and the upper aluminum layer at the midpoint is a concentrated mass. Because buckling occurs, we include the effect of the mid-plane stretching that introduces a geometric nonlinearity into the system. To develop our equations of motion we 


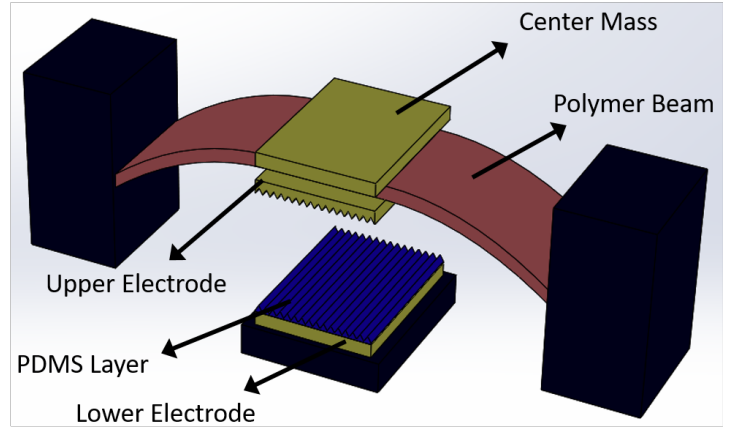

(a)

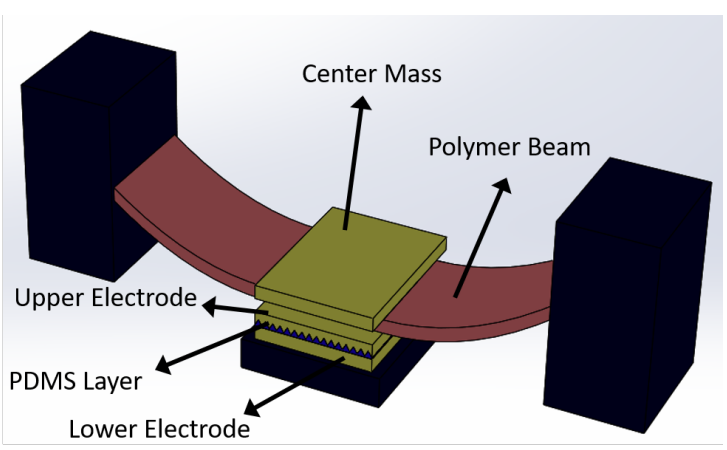

(b)

Figure 1: The working principle of the threshold shock sensor: (a) the initial buckled position held stable by the axial load; (b) the triggered position when the force from a shock exceeds a threshold and switches stable positions. The figure is not to scale.

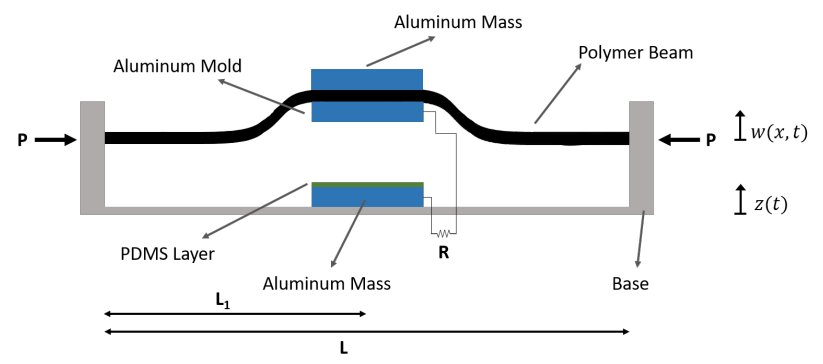

Figure 2: Schematic of shock sensor. Some features are exaggerated to help the reader visualize the system.

first describe our system. Because we are considering our system to be a beam with a concentrated mass located at the midspan, our model splits the beam into two spans.

We note that although the transverse direction is in the same direction as the gravitational force, we will neglect the effect of the gravitational force in the model because the static configurations of the system are dominated by the buckling phenomenon and not by gravity. The only parameter that would be changed by including the effect of gravity in the model is the threshold acceleration. As this study is only a proof of concept and a learning tool for the dynamics and physics of the system, we are only concerned with whether a switching event occurs. Finding the exact threshold acceleration is not the driving factor for this study. At this point, we are only concerned with demonstrating the feasibility of a triboelectric threshold shock sensor.

\subsection{Deriving Equation of Motions}

The equations of motions of this system will be derived using the Extended Hamilton's Principle, and we start by representing the system in terms of the absolute deflection of the beam and the base motion. The kinetic energy of the system can be written as

$$
T=\frac{1}{2} \rho A \int_{0}^{L_{1}} \dot{w}_{1}^{2} d x+\frac{1}{2} \rho A \int_{L_{1}}^{L} \dot{w}_{2}^{2} d x+\frac{1}{2} M \dot{w}_{1}^{2}\left(L_{1}\right)+\frac{1}{2} \rho A \int_{0}^{L_{1}} \dot{u}_{1}^{2} d x+\frac{1}{2} \rho A \int_{L_{1}}^{L} \dot{u}_{2}^{2} d x
$$

The potential energy is expressed as

$$
U=\frac{1}{2} \int_{V} \varepsilon_{s} \sigma_{s} d V+\frac{\varepsilon_{0}}{2} \int_{V} E_{a i r}^{2} d V+\frac{\varepsilon_{0} \varepsilon_{r}}{2} \int_{V} E_{P D M S}^{2} d V
$$


where

$$
\begin{aligned}
\varepsilon_{s} & =u_{i}^{\prime}+\frac{1}{2} w_{i}^{\prime^{2}}-z_{c} w_{i}^{\prime \prime} \\
\sigma_{s} & =E \varepsilon_{s} \\
E_{a i r} & =\frac{(-q+\sigma S)}{\varepsilon_{0} S} \\
E_{P D M S} & =-\frac{q}{\varepsilon_{0} \varepsilon_{r} S}
\end{aligned}
$$

Thus, the potential energy can be rewritten as

$$
\begin{aligned}
U= & \frac{1}{2} E I \int_{0}^{L_{1}} w_{1}^{\prime \prime^{2}} d x+\frac{1}{2} E I \int_{L_{1}}^{L} w_{2}^{\prime \prime^{2}} d x+\frac{E A}{2} \int_{0}^{L_{1}}\left[u_{1}^{\prime}+\frac{1}{2} w_{1}^{\prime^{2}}\right]^{2} d x+\frac{E A}{2} \int_{L_{1}}^{L}\left[u_{2}^{\prime}+\frac{1}{2} w_{2}^{\prime^{2}}\right]^{2} d x \\
& +\frac{T_{P D M S}}{2 \varepsilon_{0} \varepsilon_{r} S} q^{2}+\frac{d_{0}+w_{1}\left(L_{1}\right)-z(t)}{2 \varepsilon_{0} S} q^{2}-\frac{\sigma\left(d_{0}+w_{1}\left(L_{1}\right)-z(t)\right)}{\varepsilon_{0}} q+\frac{\sigma^{2} S\left(d_{0}+w_{1}\left(L_{1}\right)-z(t)\right)}{2 \varepsilon_{0}}
\end{aligned}
$$

The variation of the work caused by the nonconservative forces is

$$
\delta W_{n c}=-c \int_{0}^{L_{1}} \dot{w}_{1} \delta w_{1} d x-c \int_{L_{1}}^{L} \dot{w}_{2} \delta w_{2} d x-R \dot{q} \delta q+p u_{1}(0)-p u_{2}(L)
$$

Now that the system has been fully represented in terms of the absolute deflection, we restate Eqs. (1), (4) and (5) in terms of the relative motion, $y_{i}(x, t)$, such that: $w_{i}(x, t)=y_{i}(x, t)+z(t)$. The Extended Hamilton's Principle, $\int_{t_{1}}^{t_{2}}\left(\delta T-\delta U+\delta W_{n c}\right) d t=0$ is performed, which results in the governing equations for the system:

$$
\begin{aligned}
& \left\{\begin{array}{l}
\rho A \ddot{y_{1}}+\rho A \ddot{z}+c \dot{y_{1}}+E I y_{1}^{\prime \prime \prime \prime}-E A\left[\left(u_{1}^{\prime}+\frac{1}{2} y_{1}^{\prime 2}\right) y_{1}^{\prime}\right]^{\prime}=0 \quad 0 \leq x \leq L_{1} \\
\rho A \ddot{y_{2}}+\rho A \ddot{z}+c \dot{y_{2}}+E I y_{2}^{\prime \prime \prime \prime}-E A\left[\left(u_{2}^{\prime}+\frac{1}{2} y_{2}^{\prime 2}\right) y_{2}^{\prime}\right]^{\prime}=0 \quad L_{1} \leq x \leq L
\end{array}\right. \\
& \left\{\begin{array}{l}
\rho A \ddot{u_{1}}-E A\left[u_{1}^{\prime}+\frac{1}{2} y_{1}^{\prime 2}\right]^{\prime}=0 \quad 0 \leq x \leq L_{1} \\
\rho A \ddot{u_{2}}-E A\left[u_{2}^{\prime}+\frac{1}{2} y_{2}^{\prime 2}\right]^{\prime}=0 \quad L_{1} \leq x \leq L \\
\dot{q}=\frac{-q}{\varepsilon_{0} R S}\left[\frac{T_{P D M S}}{\varepsilon_{r}}+d_{0}+y_{1}\left(L_{1}\right)\right]+\frac{\sigma\left(d_{0}+y_{1}\left(L_{1}\right)\right)}{\varepsilon_{0} R}
\end{array}\right.
\end{aligned}
$$

From Eq. (6), we see that we have a coupled set of equations with the coupling between the longitudinal and transverse directions of motion. In Appendix A.1, we provide the derivation that decouples the mechanical equations of motion for the longitudinal and transverse directions. Substituting Eq. (21) into the transverse equation of motion in Eq. (6), we get 


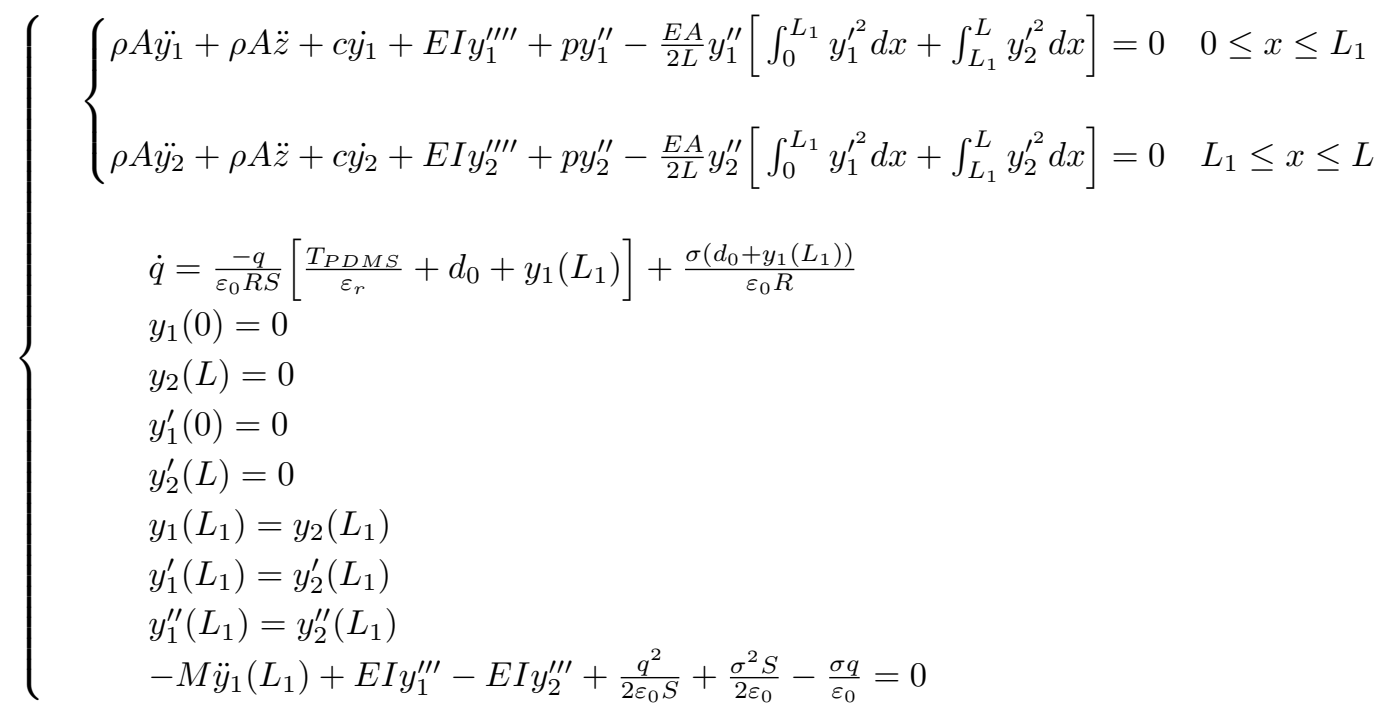

\subsection{Static Buckled Configuration}

To determine the static solution of the system, we set the time dependent variables in Eq. (7) to zero. We neglect all electrical terms because the static configuration is dependent only on the mechanical system. We note that the static configuration of the system will be the trivial solution unless the compressive axial load is greater than the first critical axial load. In determining the static configuration and the mode shapes, we will be following similar procedures from [40]. Letting the static solution be $y_{i}(x, t)=\psi_{i}(x)$ results in the static equilibrium equation and boundary conditions of

$$
\begin{aligned}
& \begin{cases}E I \psi_{1}^{\prime \prime \prime \prime}+p \psi_{1}^{\prime \prime}-\frac{E A}{2 L} \psi_{1}^{\prime \prime}\left[\int_{0}^{L_{1}} \psi_{1}^{\prime^{2}} d x+\int_{L_{1}}^{L} \psi_{2}^{\prime^{2}} d x\right]=0 & 0 \leq x \leq L_{1} \\
E I \psi_{2}^{\prime \prime \prime \prime}+p \psi_{2}^{\prime \prime}-\frac{E A}{2 L} \psi_{2}^{\prime \prime}\left[\int_{0}^{L_{1}} \psi_{1}^{\prime^{2}} d x+\int_{L_{1}}^{L} \psi_{2}^{\prime^{2}} d x\right]=0 & L_{1} \leq x \leq L\end{cases} \\
& \left\{\begin{array}{l}
\psi_{1}(0)=0 \\
\psi_{1}^{\prime}(0)=0 \\
\psi_{2}(L)=0 \\
\psi_{2}^{\prime}(L)=0 \\
\psi_{1}\left(L_{1}\right)=\psi_{2}\left(L_{1}\right) \\
\psi_{1}^{\prime}\left(L_{1}\right)=\psi_{2}^{\prime}\left(L_{1}\right) \\
\psi_{1}^{\prime \prime}\left(L_{1}\right)=\psi_{2}^{\prime \prime}\left(L_{1}\right) \\
\psi_{1}^{\prime \prime \prime}\left(L_{1}\right)=\psi_{2}^{\prime \prime \prime}\left(L_{1}\right)
\end{array}\right.
\end{aligned}
$$

This is an eigenvalue problem with eigenvalues

$$
\lambda^{2}=\frac{p}{E I}-\frac{A}{2 I L}\left[\int_{0}^{L_{1}} \psi_{1}^{\prime^{2}} d x+\int_{L_{1}}^{L} \psi_{2}^{\prime^{2}} d x\right]
$$

Therefore, the solution takes the form of

$$
\begin{aligned}
& \psi_{1}(x)=B_{1}+B_{2} x+B_{3} \cos \lambda x+B_{4} \sin \lambda x \\
& \psi_{2}(x)=B_{5}+B_{6} x+B_{7} \cos \lambda x+B_{8} \sin \lambda x
\end{aligned}
$$


By using Eq. (11) with the associated boundary conditions, a coefficient matrix is found. The first eigenvalue is obtained by equating the determinant of the coefficient matrix to zero. As we had nine unknowns and eight of them were determined by using all but one of the boundary conditions, we still have one unknown constant. After an axial force (that is beyond the critical axial load) has been chosen, we use the last constraint, Eq. (10) to determine the last unknown constant to fully determine the static buckled configuration. Now the mode shapes around the first buckled configuration can be determined. The details concerning the mode shape analysis are included in Appendix A.2.

\subsection{Reduced Order Model}

Now that the mode shape about the buckled configuration has been obtained, we can convert our set of partial differential equations into a set of ordinary differential equations. We start by defining the Lagrangian $(\mathcal{L}=T-U)$ of the system. We will simplify our notation to denote the static buckled configuration as $\psi(x)$ and the first mode shape as $\phi(x)$ and will not continue the analysis using the two span approach as the buckled configuration and mode shape are now determined. Because we have a large and rigid mass located at the center of the beam, the anti-symmetric modes are prevented. A similar phenomenon was observed for doubly clamped beams with a central clamp [41].

$$
\begin{aligned}
\mathcal{L} & =\frac{1}{2} \rho A \int_{0}^{L}(\dot{y}+\dot{z})^{2} d x+\frac{1}{2} M\left(\dot{y}\left(L_{1}\right)+\dot{z}\right)^{2}-\frac{1}{2} E I \int_{0}^{L} y^{\prime \prime^{2}} d x-\frac{E A L}{2}\left[-\frac{p}{E A}+\frac{1}{2 L} \int_{0}^{L} y^{\prime^{2}} d x\right]^{2} \\
& -\frac{T_{P D M S}}{2 \varepsilon_{0} \varepsilon_{r} S} q^{2}-\frac{d_{0}+y\left(L_{1}\right)}{2 \varepsilon_{0} S} q^{2}+\frac{\sigma\left(d_{0}+y\left(L_{1}\right)\right)}{\varepsilon_{0}} q-\frac{\sigma^{2} S\left(d_{0}+y\left(L_{1}\right)\right)}{2 \varepsilon_{0}}
\end{aligned}
$$

As we are only interested in modeling the first buckling mode, a one-mode approximation for the Galerkin discretization is used. To approximate the dynamic motion about the first buckled configuration, let

$$
y(x, t)=\psi(x)+\phi(x) \eta(t)
$$

where $\phi(x)$ is the first buckling mode shape. Substituting Eq. (13) into Eq. (12) and performing Lagrange's Equation for the variables $\eta(t)$ and $q(t)$, and adding damping result in a coupled set of differential equations with respect to $\eta(t)$ and $q(t)$. When we drop the static terms in the mechanical equation, we arrive at the dynamic coupled equations.

$$
\begin{aligned}
& M_{1} \ddot{\eta}+M_{Z} \ddot{z}+D_{1} \dot{\eta}+K_{L} \eta+K_{Q} \eta^{2}+K_{C} \eta^{3}+\alpha_{1} q^{2}+\alpha_{2} q=0 \\
& \dot{q}=-\frac{q}{\varepsilon_{0} S R}\left[\frac{T_{P D M S}}{\varepsilon_{r}}+d_{0}+\psi\left(L_{1}\right)+\phi\left(L_{1}\right) \eta\right]+\frac{\sigma\left(d_{0}+\psi\left(L_{1}\right)+\phi\left(L_{1}\right) \eta\right)}{\varepsilon_{0} R}
\end{aligned}
$$


where

$$
\begin{aligned}
M_{1}= & \rho A \int_{0}^{L} \phi^{2}(x) d x+M \phi^{2}\left(L_{1}\right) \\
M_{Z}= & \rho A \int_{0}^{L} \phi(x) d x+M \phi\left(L_{1}\right) \\
D_{1}= & c \int_{0}^{L} \phi^{2}(x) d x \\
K_{L}= & E I \int_{0}^{L} \phi^{\prime \prime 2}(x) d x-p \int_{0}^{L} \phi^{\prime 2}(x) d x+\frac{E A}{L}\left(\int_{0}^{L} \psi^{\prime}(x) \phi^{\prime}(x) d x\right)^{2} \\
& +\frac{E A}{2 L} \int_{0}^{L} \psi^{\prime^{\prime}}(x) d x \int_{0}^{L} \phi^{\prime 2}(x) d x \\
K_{Q}= & \frac{3 E A}{2 L} \int_{0}^{L} \phi^{\prime 2}(x) d x \int_{0}^{L} \psi^{\prime}(x) \phi^{\prime}(x) d x \\
K_{C}= & \frac{E A}{2 L}\left(\int_{0}^{L} \phi^{\prime 2}(x) d x\right)^{2} \\
\alpha_{1}= & \frac{\phi\left(L_{1}\right)}{2 \varepsilon_{0} S} \\
\alpha_{2}= & -\frac{\sigma \phi\left(L_{1}\right)}{\varepsilon_{0}}
\end{aligned}
$$

As we can see from Eqs. (14) and (15), we have a coupled set of differential equations that describe our dynamic system about the buckled configuration. The coupling arises from $\alpha_{1} q^{2}$ and $\alpha_{2} q$ in the mechanical equation, and the terms that contain $\eta$ in the electrical equation.

\section{Stability Analysis}

Before the full impact model is considered and the dynamics of the sensor are investigated, the stability of the system is analyzed. Intuitively, we expect to see that this system has three equilibrium positions, with two of those being stable and one being unstable showing a pitchfork bifurcation. As the stability of this system is only dependent on the mechanical terms, we will neglect the electrical terms, and the electrical differential equation. After removing the forcing and damping terms, we arrive at the Jacobian of the system.

$$
J=\left[\begin{array}{cc}
-\lambda_{1} & 1 \\
-\frac{K_{L}}{M_{1}}-\frac{2 K_{Q}}{M_{1}} \eta_{e q}-\frac{3 K_{C}}{M_{1}} \eta_{e q}^{2} & -\lambda_{1}
\end{array}\right]
$$

where the $\eta_{e q}$ terms are determined by finding the equilibrium points from the mechanical equilibrium equation $\left(K_{L} \eta_{e q}+K_{Q} \eta_{e q}^{2}+K_{C} \eta_{e q}^{3}=0\right)$. By analyzing the Jacobian, one can see two stable solutions and one unstable solutions. We now compare the approximated equilibrium profiles of the beam with the exact solution obtained from Section 3.2, see Fig. 3a. As deduced from this figure, the approximate solution using one mode is close to the exact solution, but has small deviations in predicting the unstable solution.

If we repeat this procedure with different axial forces, we can create a bifurcation diagram for the system, with the axial force being the tuning parameter. From the bifurcation diagram, Fig. 3b, one sees that in addition to the pitchfork bifurcation, there is a saddle node bifurcation that the middle and bottom solutions will eventually collide and destroy each other. Ideally, there should only be a pitchfork bifurcation, but because we are only considering one mode, we are limiting the accuracy of the dynamic system [42]. However, if the axial force is not too large, the one mode solution will be a good approximation. 


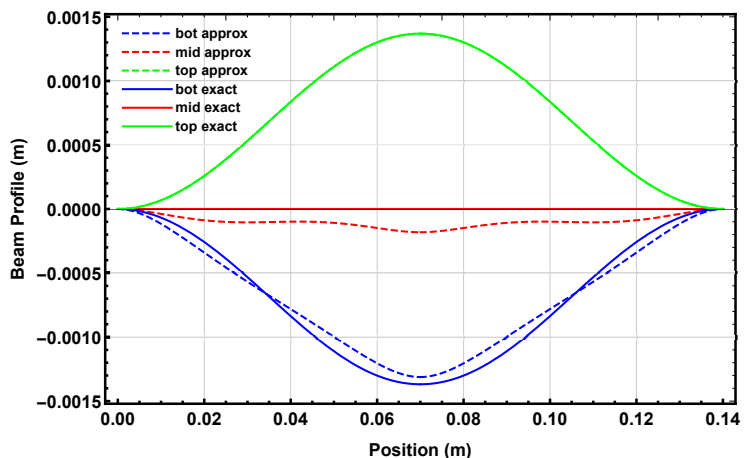

(a) Comparison between exact equilibrium beam profiles and the approximated one from the onemode Galerkin Decomposition

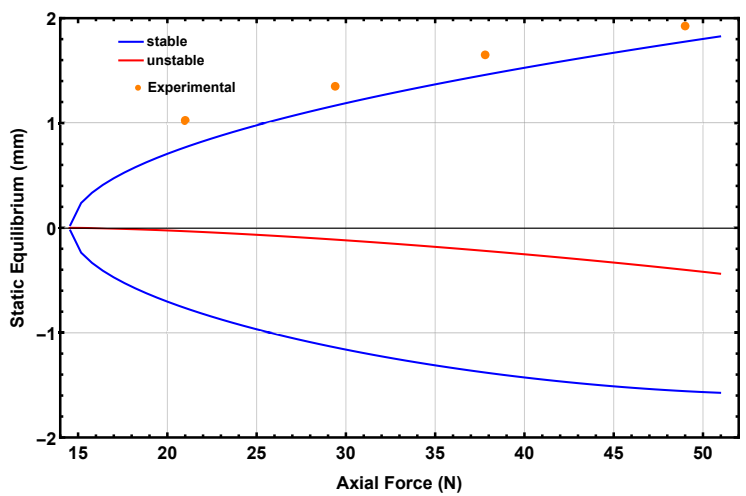

(b) Bifurcation diagram for the threshold sensor. Saddle node bifurcation is result of only considering one mode in the Galerkin Decomposition

Figure 3

\section{Impact Model}

Before the full dynamics of the system can be explored, the impact model has to be considered. Using impact equations from $[43,44]$ and adding the quadratic and cubic stiffness terms, we have

$$
\begin{aligned}
& F_{s}=k_{i} \eta+k_{i} g_{i}+K_{Q} g_{i}^{2}+K_{C} g i^{3} \\
& F_{d}=c_{i} \dot{\eta}
\end{aligned}
$$

We can now represent the full dynamic equations of motion for the free motion and the impact by using a piecewise function, with the condition being whether the upper electrode is contacting the PDMS layer.

$$
\begin{cases}M_{1} \ddot{\eta}+M_{Z} \ddot{z}+D_{1} \dot{\eta}+K_{L} \eta+K_{Q} \eta^{2}+K_{C} \eta^{3}+\alpha_{1} q^{2}+\alpha_{2} q=0 & y\left(L_{1}, t\right)>-g_{i} \\ M_{1} \ddot{\eta}+M_{Z} \ddot{z}+\left(D_{1}+c_{i}\right) \dot{\eta}+\left(K_{L}+k_{i}\right) \eta+k_{i} g_{i}+K_{Q} g_{i}^{2}+K_{C} g_{i}^{3}+\alpha_{1} q^{2}+\alpha_{2} q=0 & y\left(L_{1}, t\right) \leq-g_{i}\end{cases}
$$

where $g_{i}$ is the gap between the upper electrode and the PDMS layer. It is noted that the total initial gap, $d_{0}$, is the summation of $g_{i}$, and the penetration distance $\delta_{1}$.

\section{Experimental Setup}

To experimentally test the concept of the shock sensor, the system was placed on an electrodynamic shaker. A picture of the experimental setup and the block diagram is shown in Fig. 4. The input signal to the shaker was controlled in a closed loop with PUMA Spectral Dynamics. Once the signal was generated, the signal was sent through a power amplifier (Techron 5530 Power Supply Amplifier) and then to the shaker. For the excited base to produce a half-sine pulse, the signal to the electrodynamic shaker requires pre and post compensation pulses, Fig. 5a. These compensation pulses can be seen in Fig. 5a as the low amplitude acceleration pulses that occur before and after the main half-sine pulse. The compensation pulses allow the shaker to use more of its stroke and to produce a decent approximation to a drop table test. The input to the shaker was a half-sine wave with a frequency of $38 \mathrm{~Hz}$. An accelerometer (PCB Piezotronics 352A24) was placed on the base of the shaker and the accelerometer signal was used in the feedback control loop. The accelerometer signal was read by a data acquisition device. The voltage was recorded using an oscilloscope (Tektronix MDO3034). The axial force was controlled by adjusting a screw that was fixed to the setup, and the axial force was measured using a FlexiForce Sensor. The power amplifier that powers the electrodynamic shaker and the PUMA Spectral Dynamics system are not pictured. The system parameters are listed in Table 1. 
Because this paper is meant not only to propose a possible idea for a bi-stable threshold sensor but also to explore the physics and dynamics of the entire system so this work could be used as a future design tool, we will briefly discuss how different parameters could potentially change the system's response. One of the most important characteristics in this system is the stiffness. This is because stiffness determines the critical axial load and the acceleration threshold. Although it is unclear how the system will behave by simultaneously changing multiple parameters, we can discuss the simple cases where we change one parameter and keep the rest constant. The choice of material has a large influence because the material directly changes the stiffness of the system. A polymer beam, similar to what was used in this experiment, is much less stiff than a metal beam. By using a material with a higher modulus of elasticity, the critical buckling load will increase, and we would assume that it will take a stronger shock to switch states. This is due to the beam being able to store more energy before releasing the energy and switching states. By changing the length of the beam or the cross-sectional area, the critical load and threshold acceleration will change. Depending on which dimensions were changed, different acceleration ranges could be targeted. Concerning the electrical components, the surface area of the upper and lower electrodes and the triboelectric materials will also affect the system. With a higher charge density or contact area, larger and more noticeable voltage peaks will be created.

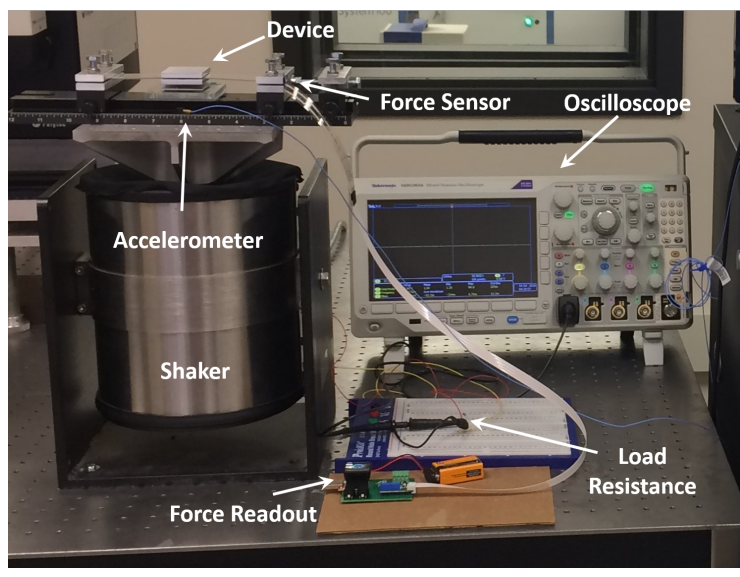

(a) Picture of the experimental setup.

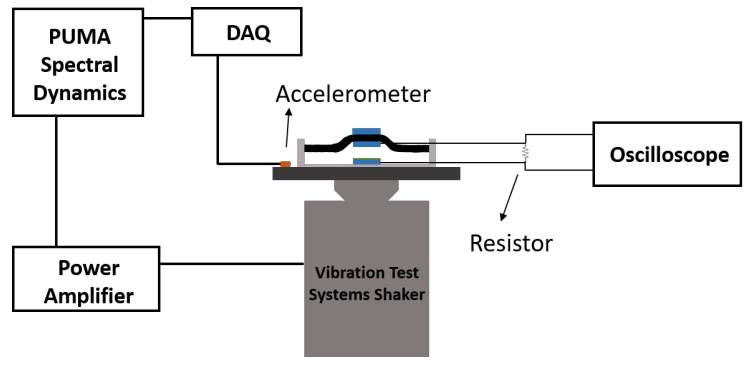

(b) Flow chart of the experimental setup.

Figure 4

\section{Results and Discussions}

Shock simulations were conducted to demonstrate the sensor response when the shock goes beyond the threshold as well as the relationship between the magnitude of the shock signal to the sensed voltage. The results are compared with the data obtained from experiments. We first present a case at an acceleration just below the threshold for this system. The input acceleration measured by an external accelerometer is shown in Fig. 5a. We note that the previously mentioned compensation pulses are not large enough to trigger the switching motion, so these pulses do not affect the system dynamics. We also note that although it looks like the base acceleration pulse and the voltage reading occur at different times, they were recorded at the same time. The difference in time comes from triggering of the oscilloscope. Figs. 5a to $5 \mathrm{c}$ show the base acceleration, the simulated voltage output, and the measured voltage output for the low acceleration case. As the threshold acceleration was not reached, the system did not switch states, which is displayed by the lack of a voltage spike. Although it may be obvious, any acceleration amplitudes lower than this low acceleration case will not trigger the switch. This design concept regarding bi-stability has the potential to be very reliable because the switching of states can only occur when the threshold acceleration is experienced. Furthermore, even if the sensor experiences an acceleration amplitude greater than the threshold value, but the direction is not in the correct orientation, the device will still not trigger. A sensor that monitors in only one plane at a time could be a drawback in some instances but very useful for systems in which the incoming shock direction is known. 


\begin{tabular}{ll} 
Parameter & Value \\
& \\
\hline$(L \times b \times t)$ & $(14 \times 3.7 \times 0.1) \mathrm{cm}$ \\
$\left(L_{m} \times b_{m} \times t_{m}\right)$ & $(3.7 \times 5.2 \times 0.45) \mathrm{cm}$ \\
$c$ & $95 \mathrm{Ns} / \mathrm{m}$ \\
$d_{0}$ & $1.3683 \mathrm{~mm}$ \\
$\delta_{1}$ & $46.40 \mu \mathrm{m}$ \\
$g_{i}$ & $1.3219 \mathrm{~mm}$ \\
$E$ & $2.344 \mathrm{GPa}$ \\
$p$ & $35 \mathrm{~N}$ \\
$R$ & $10 \mathrm{M} \Omega$ \\
$T_{P D M S}$ & $320 \mu \mathrm{m}$ \\
$\varepsilon_{r}$ & 2.5 \\
$\rho$ & $1220 \mathrm{~kg} / \mathrm{m}^{3}$ \\
$\sigma$ & variable \\
$k_{i}$ & $30000 \mathrm{~N} / \mathrm{m}$ \\
$c_{i}$ & $3000 \mathrm{Ns} / \mathrm{m}$ \\
\hline
\end{tabular}

Table 1: Experimental parameters

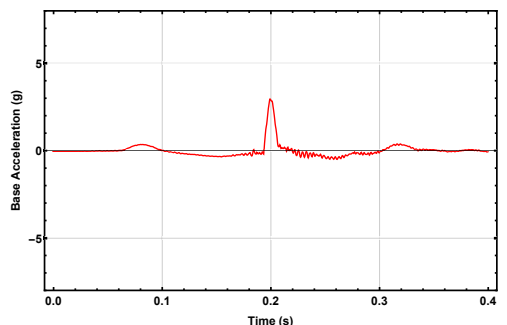

(a) Experimental results for the input acceleration pulse at $2.95 \mathrm{~g}$

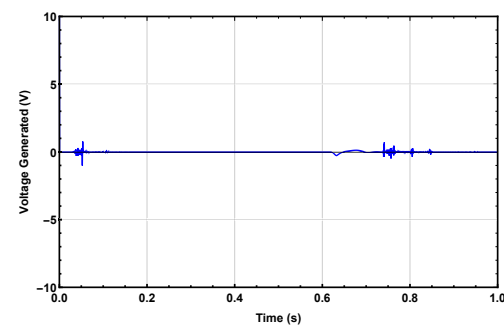

(b) Simulation of voltage response with shock amplitude of $2.95 g$ and $\sigma=4.2 \frac{\mu C}{m^{2}}$

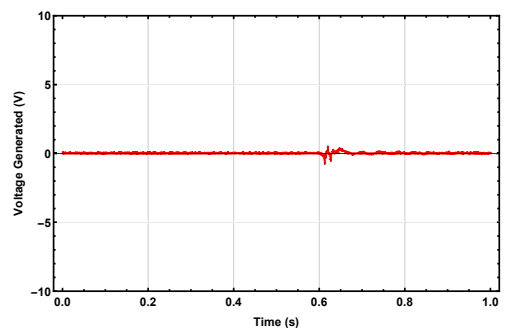

(c) Experimental voltage results for shock amplitude of $2.95 \mathrm{~g}$

Figure 5

At the threshold acceleration, Fig. 6, there is a significant voltage spike when the sensor experiences input shock. As the upper electrode is switching stable states and moving toward the lower electrode, we see a negative voltage spike. If we compare this threshold case to the previous case in Fig. 5, we see a very pronounced difference in the voltage outputs. This is one of the main advantages of this specific design, as there is a large signal-to-noise ratio when the device is triggered.

After the initial negative voltage spike, we see a positive voltage spike and then another negative voltage spike. This positive spike is due to an imperfect collision between the upper and lower electrodes. Ideally, when the electrodes come into contact with each other, the velocity of the upper electrode would immediately drop to zero and would remain at a constant zero velocity. But in reality, the upper electrode will start to rebound off of the lower electrode and impact again, which is why we see the positive spike followed by another negative spike. When we discuss the voltage peak for the experimental data we will be referring to the negative voltage peak as this peak is the first to occur and has the greatest magnitude. To further illustrate the relationship between the mechanical motion and the electrical output, we present Fig. 7a, which displays the displacement and velocity of the upper electrode (beam midspan) once the shock pulse is felt. Fig. $7 \mathrm{~b}$ displays the charge transferred between the electrodes and the voltage across the load when the shock pulse is felt. Figs. $7 \mathrm{a}$ and $7 \mathrm{~b}$ are performed under an acceleration amplitude of $4.56 \mathrm{~g}$. From Fig. 7a, when the upper electrode is crossing through the unstable equilibrium point, the maximum velocity occurs. As the upper electrode moves toward the lower electrode and the velocity changes rapidly, the charge 


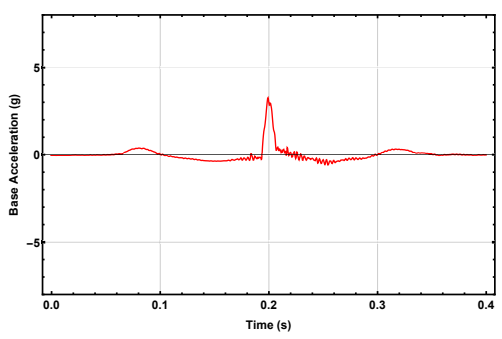

(a) Experimental results for the input acceleration pulse at $3.26 \mathrm{~g}$

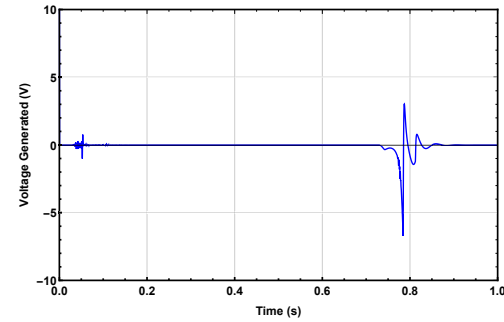

(b) Simulation of voltage response with shock amplitude of $3.26 \mathrm{~g}$ and $\sigma=4.2 \frac{\mu C}{m^{2}}$

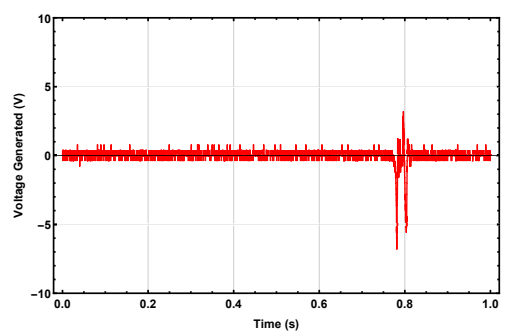

(c) Experimental voltage results for shock amplitude of $3.26 \mathrm{~g}$

Figure 6

changes quickly. This change in charge results in the negative voltage spike. Once the impact occurs, the velocity dramatically decreases in magnitude and the upper electrode rebounds slightly apart from the lower electrode and then impacts again. This small deviation is what causes the positive and second negative peak. After these three voltage peaks have occurred, the system settles into a state of equilibrium between all of these quantities.

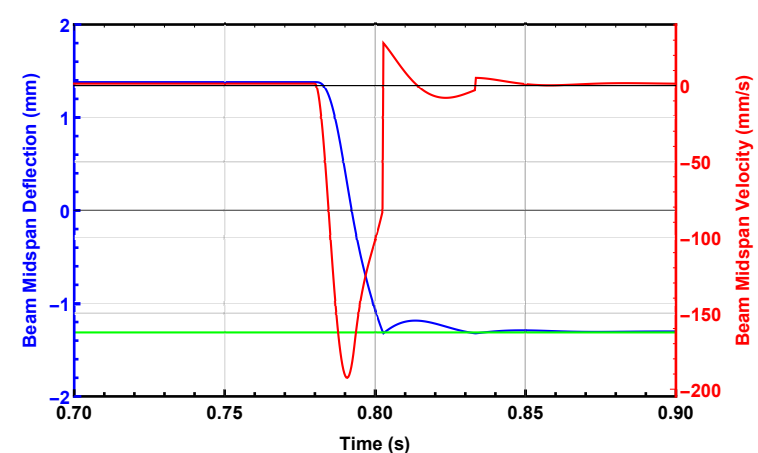

(a) Position and velocity of beam midspan for acceleration amplitude of $4.56 \mathrm{~g}$. The green line represents the position of the PDMS layer on the lower electrode.

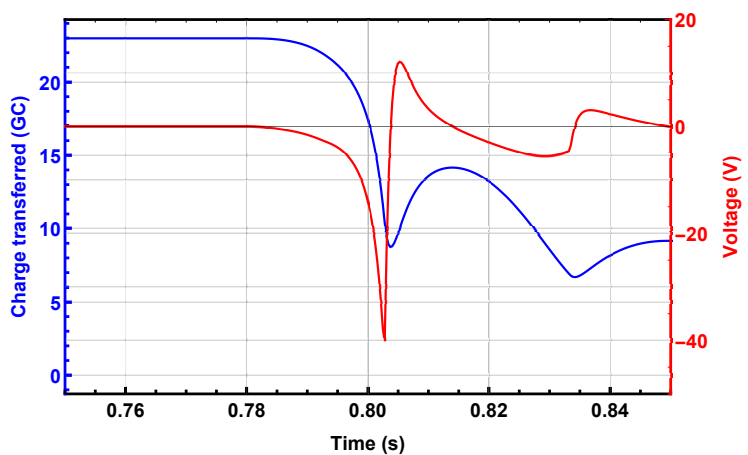

(b) Charge transferred and voltage output for acceleration amplitude of $4.56 \mathrm{~g}$

Figure 7

Next, we increase the input shock amplitude to magnitudes larger than the threshold amplitude. This is to display the characteristic of the shock sensor as an accelerometer beyond the threshold. We not only see a voltage spike that indicates that the threshold acceleration has been reached, but we see a voltage peak that is related to the magnitude of the input shock signal. As we expect to see, as the input shock amplitude increases, we see a larger negative voltage peak. These results are displayed in Figs. 8 and 9 . We see that there is a close agreement in the simulation and experimental results on the negative voltage spike that occurs from switching between the two stable states. It is noted that the surface charge density used in simulations is identified from experiments, which shows its increase from increasing the shock level. The reason is the stronger impact causes larger penetration into the PDMS layer [39], hence, more charges are generated on the contact surfaces resulting in larger surface charge density.

At last, the relationship between the input acceleration magnitude and the output voltage peak is demonstrated, see Fig. 10. Again, for the output voltage signal, we are just considering the magnitude of the negative voltage spike, as that is the voltage associated with the input shock signal. It is deduced that the sensor has zero output below the threshold shock of $3.26 \mathrm{~g}$, and its voltage output is fairly linearly proportional to the acceleration beyond the threshold as seen in Fig. 10. Although some of these voltage peaks 


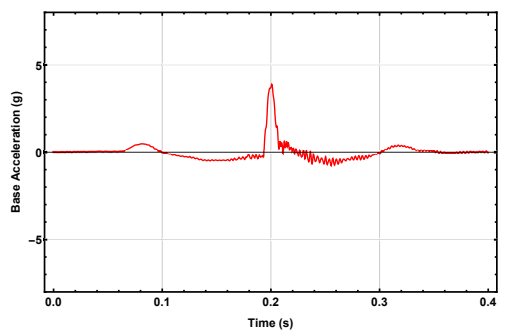

(a) Experimental results for the input acceleration pulse at $3.88 \mathrm{~g}$

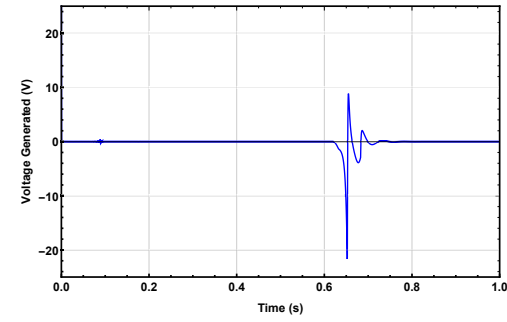

(b) Simulation of voltage response with shock amplitude of $3.88 g$ and $\sigma=10.5 \frac{\mu C}{m^{2}}$

Figure 8

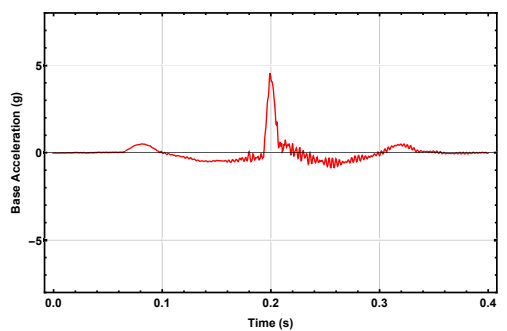

(a) Experimental results for the input acceleration pulse at $4.56 \mathrm{~g}$

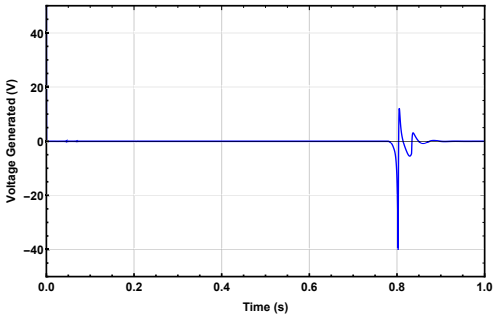

(b) Simulation of voltage response with shock amplitude of $4.56 \mathrm{~g}$ and $\sigma=12.5 \frac{\mu C}{m^{2}}$

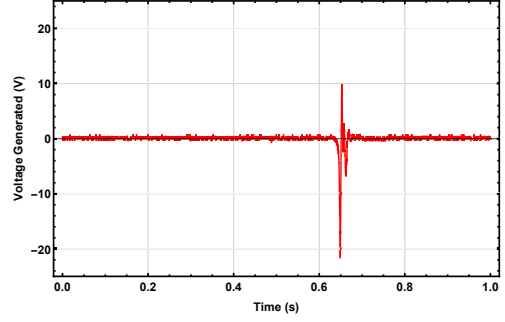

(c) Experimental voltage results for shock amplitude of $3.88 \mathrm{~g}$

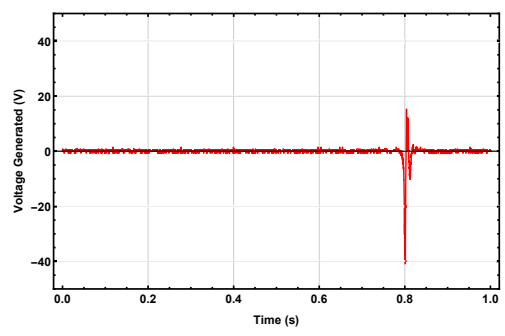

(c) Experimental voltage results for shock amplitude of $4.56 \mathrm{~g}$

Figure 9

are not monotonically increasing with the acceleration amplitude, Fig. 10 still shows that there is a general linear trend between input acceleration and voltage. As this idea is only a proof of concept, future work will be done to address the slight variations in the voltage amplitude. The results indicate the addition of a buckling mechanism to the triboelectric generator enables a threshold shock sensor that responds to accelerations beyond a threshold. In addition, the proportionality of the output voltage to the acceleration beyond the threshold reveals that a threshold sensor can effectively be used as an accelerometer.

This characterization effort is an important part of the sensor development that can be completed by adding an electrical readout. In a practical application, there will need to be a power input to relate the shock acceleration to the impact intensity, which would be done in a signal processing unit. The powering could use a button size battery that is only used when the processing unit receives a signal. Attaching a similar system to a shipping container for example would be very valuable because if the container was dropped from a certain height, the system would switch states and provide information about the intensity of the drop. At this stage though, we are not very concerned with how the system will be powered because we intended to provide an understanding of the combined phenomena of bi-stability and triboelectricity and to describe the underlying multiphysics through mathematical modeling that will be important for the development of the sensor.

As the goal for this proposed idea is for it to be used on the micro-scale, it is imperative to see if this idea can be scaled down. In the micro-scale, the axial stresses have been generated by thermal expansion induced by Joule heating that controlled the curvature of buckled beams [45-47]. The other method is based on using predefined geometries to enable bi-stability [41, 48]. Beams can be made bi-stable using predefined profiles, which gives precise control of its curvature. The predefined curvature is useful when the range of shock is fixed. If the shock range is variable, current induced thermal stresses can tune the threshold shock the sensor can detect. 


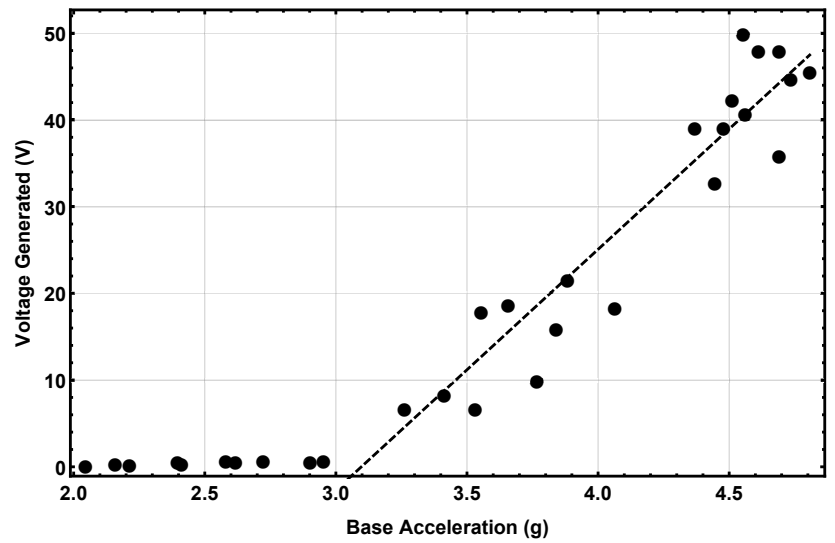

Figure 10: Experimental output voltages of the sensor as a function of shock accelerations. A linear fit is used to characterize the relationship between voltage and shock amplitude.

\section{Conclusions}

A proposed design and proof of concept of a bi-stable threshold sensor using triboelectric transduction were investigated. A continuous model using nonlinear Euler-Bernoulli beam theory was derived to accurately describe the system behavior. The model proved to accurately capture the system dynamics as well as output voltages observed experimentally. This presented sensor was not only able to display a significant voltage reading when the acceleration exceeds a threshold, but it was also able to relate the amplitude of the input shock signal to the output voltage amplitude. This is advantageous as the user would be able to know how much of a shock the device experienced during the triggering and not just that the device was triggered. This device also can be tuned by adjusting the axial force that will determine the buckling level of the beam. Increasing the axial force enhances the sensor robustness and increases the threshold shock it can detect. This capability enables a tunable shock sensor. For the parameters that were used this study, we were able to trigger the device at fairly low g-levels $(3.26 \mathrm{~g})$, which can be very useful for low shock levels. Although this specific sensor is not on the micro-scale, the fundamental understanding of the system behavior and its characterization are useful for the future development of a miniature counterpart.

\section{References}

[1] G. Shi, C.-S. Chan, G. Zhang, W. J. Li, P. H. Leong, K.-S. Leung, Towards a mobile airbag system using mems sensors and embedded intelligence, in: Robotics and Biomimetics, 2007. ROBIO 2007. IEEE International Conference on, 2007, pp. 634-639.

[2] J. S. Go, Y. H. Cho, B. M. Kwak, K. Park, Snapping microswitches with adjustable acceleration threshold, Sensors and Actuators, A: Physical 54 (1-3) (1996) 579-583.

[3] J. Zhao, J. Jia, H. Wang, W. Li, A novel threshold accelerometer with postbuckling structures for airbag restraint systems, IEEE Sensors Journal 7 (8) (2007) 1102-1109.

[4] A. Ramini, M. I. Younis, Q. T. Su, A low-g electrostatically actuated resonant switch, Smart Materials and Structures 22 (2) (2012) 025006.

[5] S. Michaelis, H. J. Timme, M. Wycisk, J. Binder, Additive electroplating technology as a post-CMOS process for the production of MEMS acceleration-threshold switches for transportation applications, Journal of Micromechanics and Microengineering 10 (2) (2000) 120-123.

[6] T. Matsunaga, M. Esashi, Acceleration switch with extended holding time using squeeze film effect for side airbag systems, Sensors and Actuators, A: Physical 100 (1) (2002) 10-17. 
[7] L. Zimmermann, J. Ebersohl, F. L. Hung, J. Berry, F. Baillieu, P. Rey, B. Diem, S. Renard, P. Caillat, Airbag application: a microsystem including a silicon capacitive accelerometer, cmos switched capacitor electronics and true self-test capability, Sensors and Actuators A: Physical 46 (1) (1995) 190 - 195.

[8] I. D. A. Woodman, R. Michael, United States Patent ( 19 ) (19).

[9] H. Gleason, $32.4 \%-4 \%$ (1953) 2-7.

[10] A. Ibrahim, M. I. Younis, Simple fall criteria for MEMS sensors: Data analysis and sensor concept, Sensors (Switzerland) 14 (7) (2014) 12149-12173.

[11] L. J. Currano, M. Yu, B. Balachandran, Latching in a mems shock sensor: Modeling and experiments, Sensors and Actuators A: Physical 159 (1) (2010) $41-50$.

[12] L. J. Currano, S. Bauman, W. Churaman, M. Peckerar, J. Wienke, S. Kim, M. Yu, B. Balachandran, Latching ultra-low power mems shock sensors for acceleration monitoring, Sensors and Actuators A: Physical 147 (2) (2008) 490 - 497.

[13] I.-H. Hwang, Y.-S. Shim, J.-H. Lee, Modeling and experimental characterization of the chevron-type bi-stable microactuator, Journal of Micromechanics and Microengineering 13 (6) (2003) 948.

[14] J. Casals-Terre, A. Shkel, Dynamic analysis of a snap-action micromechanism, in: Sensors, 2004. Proceedings of IEEE, IEEE, 2004, pp. 1245-1248.

[15] H. N. Kwon, I.-H. Hwang, J.-H. Lee, A pulse-operating electrostatic microactuator for bi-stable latching, Journal of Micromechanics and Microengineering 15 (8) (2005) 1511.

[16] S. Krylov, B. R. Ilic, D. Schreiber, S. Seretensky, H. Craighead, The pull-in behavior of electrostatically actuated bistable microstructures, Journal of Micromechanics and Microengineering 18 (5) (2008) 055026 .

[17] J. Qiu, J. H. Lang, A. H. Slocum, A. C. Weber, A bulk-micromachined bistable relay with u-shaped thermal actuators, Journal of Microelectromechanical Systems 14 (5) (2005) 1099-1109.

[18] D. L. Wilcox, L. L. Howell, Fully compliant tensural bistable micromechanisms (ftbm), Journal of Microelectromechanical Systems 14 (6) (2005) 1223-1235.

[19] Y.-J. Yang, B.-T. Liao, W.-C. Kuo, A novel $2 \times 2$ mems optical switch using the split cross-bar design, Journal of Micromechanics and Microengineering 17 (5) (2007) 875.

[20] M. Ueda, Y. Kaneko, Y. Nishitani, A. Omote, Battery-less shock-recording device consisting of a piezoelectric sensor and a ferroelectric-gate field-effect transistor, Sensors and Actuators A: Physical 232 (2015) $75-83$.

[21] G. Giannopoulos, J. Monreal, J. Vantomme, Snap-through buckling behavior of piezoelectric bimorph beams: I. analytical and numerical modeling, Smart materials and structures 16 (4) (2007) 1148.

[22] H.-C. Lee, J.-H. Park, J.-Y. Park, H.-J. Nam, J.-U. Bu, Design, fabrication and rf performances of two different types of piezoelectrically actuated ohmic mems switches, Journal of micromechanics and microengineering 15 (11) (2005) 2098.

[23] J.-H. Park, H.-C. Lee, Y.-H. Park, Y.-D. Kim, C.-H. Ji, J. Bu, H.-J. Nam, A fully wafer-level packaged rf mems switch with low actuation voltage using a piezoelectric actuator, Journal of Micromechanics and Microengineering 16 (11) (2006) 2281.

[24] S. Gross, S. Tadigadapa, T. Jackson, S. Trolier-McKinstry, Q. Zhang, Lead-zirconate-titanate-based piezoelectric micromachined switch, Applied Physics Letters 83 (1) (2003) 174-176.

[25] R. G. Polcawich, J. S. Pulskamp, D. Judy, P. Ranade, S. Trolier-McKinstry, M. Dubey, Surface Micromachined Microelectromechancial Ohmic Series Switch Using Thin-Film Piezoelectric Actuators, IEEE Transactions on Microwave Theory and Techniques 55 (12) (2007) 2642-2654. 
[26] M. Sulfridge, T. Saif, N. Miller, K. O'Hara, Optical actuation of a bistable mems, Journal of microelectromechanical systems 11 (5) (2002) 574-583.

[27] J. S. Ko, M. G. Lee, J. S. Han, J. S. Go, B. Shin, D.-S. Lee, A laterally-driven bistable electromagnetic microrelay, ETRI journal 28 (3) (2006) 389-392.

[28] A. Cao, J. Kim, L. Lin, Bi-directional electrothermal electromagnetic actuators, Journal of Micromechanics and Microengineering 17 (5) (2007) 975.

[29] J. Casals-Terre, A. Fargas-Marques, A. M. Shkel, Snap-action bistable micromechanisms actuated by nonlinear resonance, Journal of microelectromechanical systems 17 (5) (2008) 1082-1093.

[30] W. Kreider, A. H. Nayfeh, Experimental investigation of single-mode responses in a fixed-fixed buckled beam, Nonlinear Dynamics 15 (2) (1998) 155-177.

[31] K. Das, R. Batra, Pull-in and snap-through instabilities in transient deformations of microelectromechanical systems, Journal of Micromechanics and Microengineering 19 (3) (2009) 035008.

[32] M. Vangbo, An analytical analysis of a compressed bistable buckled beam, Sensors and Actuators, A: Physical 69 (3) (1998) 212-216.

[33] J.-S. Chen, J.-S. Lin, Exact Critical Loads for a Pinned Half-Sine Arch Under End Couples, Journal of Applied Mechanics 72 (1) (2005) 147.

[34] C. Robinson, D. Overman, R. Warner, T. Blomqulst, Problems encountered in the development of a microscale g-switch using three design approaches, in: Proc. Int. Conf. on Solid-State Sensors and Actuators, Tokyo, Japan, 1987, pp. 410-413.

[35] Y. Loke, G. McKinnon, M. Brett, Fabrication and characterization of silicon micromachined threshold accelerometers, Sensors and Actuators A: Physical 29 (3) (1991) 235-240.

[36] T. Tønnesen, O. Lüdtke, J. Noetzel, J. Binder, G. Mader, Simulation, design and fabrication of electroplated acceleration switches, Journal of Micromechanics and Microengineering 7 (3) (1997) 237-239.

[37] P. Man, C. Mastrangelo, Surface micromachined shock sensor for impact detection, in: Solid-State Sensor and Actuato Workshop (Hilton Head), 1994, pp. 156-9.

[38] C. Zhang, T. Zhou, W. Tang, C. Han, L. Zhang, Z. L. Wang, Rotating-disk-based direct-current triboelectric nanogenerator, Advanced Energy Materials 4 (9).

[39] A. Ibrahim, A. Ramini, S. Towfighian, Experimental and theoretical investigation of an impact vibration harvester with triboelectric transduction, Journal of Sound and Vibration 416 (2018) 111-124.

[40] A. H. Nayfeh, P. F. Pai, Linear and nonlinear structural mechanics, New York ; Chichester : Wiley, 2004, formerly CIP.

[41] A. Frangi, B. D. Masi, F. Confalonieri, S. Zerbini, Threshold shock sensor based on a bistable mechanism: Design, modeling, and measurements, Journal of Microelectromechanical Systems 24 (6) (2015) 20192026.

[42] S. A. Emam, A theoretical and experimental study of nonlinear dynamics of buckled beams, dissertation, Virginia Polytechnic Institute and State University (2002).

[43] A. Narimani, M. E. Golnaraghi, G. N. Jazar, Frequency response of a piecewise linear vibration isolator, Modal Analysis 10 (12) (2004) 1775-1794.

[44] M. A. E. Mahmoud, E. M. Abdel-Rahmany, E. F. El-Saadany, R. R. Mansour, Battery-less electrostatic micro-power generator, in: 2009 2nd Microsystems and Nanoelectronics Research Conference, 2009, pp. $29-32$.

[45] A. Z. Hajjaj, N. Alcheikh, A. Ramini, M. A. Al Hafiz, M. I. Younis, Highly tunable electrothermally and electrostatically actuated resonators, Journal of Microelectromechanical Systems 25 (3) (2016) 440-449. 
[46] A. Z. Hajjaj, A. Ramini, N. Alcheikh, M. I. Younis, Electrothermally tunable arch resonator, Journal of Microelectromechanical Systems 26 (4) (2017) 837-845.

[47] N. Alcheikh, A. Z. Hajjaj, N. Jaber, M. I. Younis, Electrothermally actuated tunable clamped-guided resonant microbeams, Mechanical Systems and Signal Processing 98 (2018) 1069-1076.

[48] J. Qiu, J. H. Lang, A. H. Slocum, A Curved-Beam Bistable Mechanism 13 (2) (2004) 137-146.

\section{Appendix A}

\section{A.1. Decoupling Longitudinal and Transverse Equations of Motion}

To decouple the transverse and longitudinal equations of motion, we first consider the longitudinal equations of motion. We assume that the longitudinal inertia term is negligible. Using Eq. (6) and neglecting the longitudinal inertia, we develop the governing equations for the longitudinal direction, as well as the boundary conditions from the Extended Hamilton's Principle.

$$
\left\{\begin{array}{l}
u_{1}(x)=\gamma_{1}(t) x+\gamma_{2}(t)-\int_{0}^{x} \frac{1}{2} y_{1}^{\prime^{2}} d x \\
u_{2}(x)=\gamma_{3}(t)\left(x-L_{1}\right)+\gamma_{4}(t)-\int_{L_{1}}^{x} \frac{1}{2} y_{2}^{\prime^{2}} d x \\
u_{1}(0)=\frac{p L_{1}}{E A} \\
u_{2}(L)=-\frac{p\left(L-L_{1}\right)}{E A} \\
u_{1}\left(L_{1}\right)=u_{2}\left(L_{1}\right) \\
E A\left[u_{1}^{\prime}\left(L_{1}\right)+\frac{1}{2} y_{1}^{\prime^{2}}\left(L_{1}\right)\right]=E A\left[u_{2}^{\prime}\left(L_{1}\right)+\frac{1}{2} y_{2}^{\prime^{2}}\left(L_{1}\right)\right]
\end{array}\right.
$$

To further simplify, we note that while expanding the governing equations for the longitudinal direction, we determined that

$$
\begin{aligned}
& u_{1}^{\prime}(x)+\frac{1}{2} y_{1}^{\prime^{2}}(x)=\gamma_{1}(t) \\
& u_{2}^{\prime}(x)+\frac{1}{2} y_{2}^{\prime 2}(x)=\gamma_{3}(t)
\end{aligned}
$$

Now based on the last part of Eq. (19) and Eq. (20), we conclude that $\gamma_{1}(t)=\gamma_{3}(t)$. Using this simplification and the rest of the boundary conditions, we determine that

$$
\gamma_{1}(t)=\gamma_{3}(t)=-\frac{p}{E A}+\frac{1}{2 L} \int_{0}^{L_{1}} y_{1}^{\prime 2} d x+\frac{1}{2 L} \int_{L_{1}}^{L} y_{2}^{\prime 2} d x
$$

\section{A.2. Mode Shape Analysis}

The mode shapes and natural frequencies of the system around the buckled configuration are determined next. Because we are concerned with the vibrations around the buckled configuration, we let

$$
y_{i}(x, t)=\psi_{i}(x)+v_{i}(x, t)
$$

Eq. (22) is then substituted into the beam equations of motion in Eq. (7) and the forcing and damping terms are neglected. It is further simplified by using the static equations of motion in Eq. (8) and by only retaining the linear terms in $v_{i}(x, t)$ :

$$
\begin{cases}\frac{\rho A}{E I} \ddot{v}_{1}+v_{1}^{\prime \prime \prime \prime}+\lambda^{2} v_{1}^{\prime \prime}-\frac{A}{2 I L} \psi_{1}^{\prime \prime}\left[\int_{0}^{L_{1}} \psi_{1}^{\prime} v_{1}^{\prime} d x+\int_{L_{1}}^{L} \psi_{2}^{\prime} v_{2}^{\prime} d x\right]=0 & 0 \leq x \leq L_{1} \\ \frac{\rho A}{E I} \ddot{v}_{2}+v_{2}^{\prime \prime \prime \prime}+\lambda^{2} v_{2}^{\prime \prime}-\frac{A}{2 I L} \psi_{2}^{\prime \prime}\left[\int_{0}^{L_{1}} \psi_{1}^{\prime} v_{1}^{\prime} d x+\int_{L_{1}}^{L} \psi_{2}^{\prime} v_{2}^{\prime} d x\right]=0 & L_{1} \leq x \leq L\end{cases}
$$


Let $v_{i}=\phi_{i}(x) e^{j \omega t}, \alpha=\lambda^{2}$, and $\beta=\frac{\rho A \omega^{2}}{E I}$. Based on Eq. (23), the mode shape will be represented by a homogeneous solution and a particular solution, with the entire mode shape taking the form of

$$
\phi_{i}(x)=B_{1} \sinh s_{1} x+B_{2} \cosh s_{1} x+B_{3} \sin s_{2} x+B_{4} \cos s_{2} x+B_{5} \psi_{i}^{\prime \prime}(x)
$$

where $s_{1,2}=\sqrt{\frac{\mp \alpha+\sqrt{\alpha^{2}+4 \beta}}{2}}$. 